TITLE:

\title{
Cylindrical Couette flow of a rarefied gas: Effect of a boundary condition on the inverted velocity profile.
}

AUTHOR(S):

Kosuge, Shingo

CITATION:

Kosuge, Shingo. Cylindrical Couette flow of a rarefied gas: Effect of a boundary condition on the inverted velocity profile.. Physical review. E, Statistical, nonlinear, and soft matter physics 2015, 92(1): 013013.

\section{ISSUE DATE:}

2015-07

URL:

http://hdl.handle.net/2433/201553

RIGHT:

C)2015 American Physical Society 


\title{
Cylindrical Couette flow of a rarefied gas: Effect of a boundary condition on the inverted velocity profile
}

\author{
Shingo Kosuge* \\ Department of Mechanical Engineering and Science and Advanced Research Institute of Fluid Science and Engineering, \\ Graduate School of Engineering, Kyoto University, Kyoto 615-8540, Japan
}

(Received 30 March 2015; published 13 July 2015)

\begin{abstract}
The cylindrical Couette flow of a rarefied gas between a rotating inner cylinder and a stationary outer cylinder is investigated under the following two kinds of kinetic boundary conditions. One is the modified Maxwell-type boundary condition proposed by Dadzie and Méolans [J. Math. Phys. 45, 1804 (2004)] and the other is the Cercignani-Lampis condition, both of which have separate accommodation coefficients associated with the molecular velocity component normal to the boundary and with the tangential component. An asymptotic analysis of the Boltzmann equation for small Knudsen numbers and a numerical analysis of the Bhatnagar-Gross-Krook model equation for a wide range of the Knudsen number are performed to clarify the effect of each accommodation coefficient as well as of the boundary condition itself on the behavior of the gas, especially on the flow-velocity profile. As a result, the velocity-slip and temperature-jump conditions corresponding to the above kinetic boundary conditions are derived, which are necessary for the fluid-dynamic description of the problem for small Knudsen numbers. The parameter range for the onset of the velocity inversion phenomenon, which is related mainly to the decrease in the tangential momentum accommodation, is also obtained.
\end{abstract}

DOI: 10.1103/PhysRevE.92.013013

PACS number(s): 47.45.Gx, 51.10.+y, 05.20.Dd

\section{INTRODUCTION}

The cylindrical Couette flow is one of fundamental problems in fluid mechanics. If the inner cylinder is rotating at constant speed whereas the outer one is at rest, the circumferential flow speed will usually take its maximum on the inner cylinder and decrease monotonically to its minimum on the outer cylinder. In the case of a rarefied gas, however, the velocity slip on the boundaries may induce a nonmonotonic velocity profile; as the accommodation of the gas to the outer cylinder becomes weak, the slip on the outer cylinder grows and the velocity profile between the cylinders may have an inverted gradient. This phenomenon was first recognized in Ref. [1] and has been studied by subsequent analyses based on the Boltzmann equation with the Maxwell-type (diffuse-specular) boundary condition [2-4], those based on the (incompressible) Navier-Stokes equation [5-10] where the effect of rarefaction was taken into account via various velocity-slip models, and so forth [11,12].

In the present study, we investigate the same problem on the basis of kinetic theory employing the following two boundary conditions on the cylinders: we employ (i) a modified Maxwell-type condition [13] (see also Refs. [14,15]) and (ii) the Cercignani-Lampis (CL) condition [16]. Both of those conditions have the flexibility to adjust the tangential momentum accommodation coefficient (TMAC) independently of another accommodation coefficient concerning the molecular velocity component normal to the boundary. By using them, we aim to distinguish the effect of each accommodation coefficient on the behavior of the gas, especially on the flow-velocity profile, more clearly than in the case of the (standard) Maxwell-type condition employed in the previous studies [2-4].

The paper is organized as follows. After the formulation of the problem in Sec. II, we first carry out an asymptotic analysis

*kosuge.shingo.6r@kyoto-u.ac.jp of the Boltzmann equation for small Knudsen numbers in Sec. III. The velocity-slip and temperature-jump conditions on the cylinders corresponding to the above two kinetic boundary conditions are derived, which are necessary to the fluid-dynamic equation in Ref. [3]. Numerical solutions of the fluid-dynamic system thus obtained are shown, and the linearization (in the case of slow rotation of the cylinder) is performed to derive the exact solutions. Then in Sec. IV, we carry out a numerical analysis of the Bhatnagar-GrossKrook (BGK) model equation [17,18] (instead of the original Boltzmann equation) to investigate the problem for a wide range of the Knudsen number. The profiles of the macroscopic quantities and the parameter range for the onset of the velocity inversion are obtained, which confirm our expectation that the onset is related mainly to a decrease in the TMAC. Finally, the paper is concluded in Sec. V. This paper is the follow-up of Ref. [19] reported in the conference proceedings.

\section{FORMULATION}

\section{A. Problem and assumptions}

Let us consider a rarefied gas between two coaxial cylinders. The inner cylinder with radius $r_{I}$ and temperature $T_{I}$ rotates at a constant surface speed $V_{I}$, whereas the outer cylinder with radius $r_{O}$ and temperature $T_{O}$ is at rest. We investigate the steady behavior of the gas on the basis of the Boltzmann equation, restricting ourselves to an axially and circumferentially uniform case [the BGK model equation $[17,18]$ is employed in the numerical analysis in Sec. IV]. As for the boundary condition on the cylinders, we assume (i) a modified Maxwell-type condition [13] or (ii) the Cercignani-Lampis (CL) condition [16].

\section{B. Basic equation}

We first introduce the cylindrical coordinate system $(r, \theta, z)$, where the $z$ axis corresponds to the common axis of the 
cylinders. Let $\xi$ denote the molecular velocity and $\xi_{r}, \xi_{\theta}$, and $\xi_{z}$ denote, respectively, the $r, \theta$, and $z$ component of $\xi ; f(r, \xi)$ denotes the velocity distribution function (VDF) of molecules. The macroscopic quantities of the gas, such as the density $\rho$, flow velocity $\boldsymbol{v}$, pressure $p$, and temperature $T$, are defined by Eq. (1) below in terms of moments of the VDF; $v_{r}, v_{\theta}$, and $v_{z}$ denote, respectively, the $r, \theta$, and $z$ component of $\boldsymbol{v}$ ( $v_{r}=v_{z}=0$ in the present study),

$$
\begin{aligned}
& \rho=\int f d \xi, \quad \boldsymbol{v}=\frac{1}{\rho} \int \xi f d \boldsymbol{\xi}, \\
& p=R \rho T=\frac{1}{3} \int|\boldsymbol{\xi}-\boldsymbol{v}|^{2} f d \boldsymbol{\xi},
\end{aligned}
$$

where $R$ is the specific gas constant and $d \xi=d \xi_{r} d \xi_{\theta} d \xi_{z}$. The domain of integration is the whole space of $\boldsymbol{\xi}$.

The Boltzmann equation for the present problem is written as

$$
\xi_{r} \frac{\partial f}{\partial r}+\frac{\xi_{\theta}^{2}}{r} \frac{\partial f}{\partial \xi_{r}}-\frac{\xi_{r} \xi_{\theta}}{r} \frac{\partial f}{\partial \xi_{\theta}}=J,
$$

where $J$ is the collision term, the explicit form of which is omitted here (see, e.g., Refs. [20-22]). In the case of the BGK model,

$$
\begin{aligned}
J & =A \rho\left(f_{e}-f\right), \\
f_{e} & =\frac{\rho}{(2 \pi R T)^{3 / 2}} \exp \left(-\frac{|\boldsymbol{\xi}-\boldsymbol{v}|^{2}}{2 R T}\right),
\end{aligned}
$$

where $A$ is a constant and $A \rho$ corresponds to the molecular collision frequency.

\section{Boundary conditions}

The boundary condition (BC) is expressed in terms of the scattering kernel $K$ (see, e.g., Refs. [20,22]) as

$$
\begin{aligned}
f\left(r_{i}, \boldsymbol{\xi}\right)= & \int_{\bar{\xi}_{r} n_{i}<0}\left|\frac{\bar{\xi}_{r}}{\xi_{r}}\right| K(\bar{\xi}, \xi) f\left(r_{i}, \bar{\xi}\right) d \bar{\xi} \\
& \text { for } \xi_{r} n_{i}>0 .
\end{aligned}
$$

Here and in the following, the subscript $i$ represents $I$ (the inner cylinder) or $O$ (the outer cylinder); $n_{I}=1, n_{O}=-1$, and $d \bar{\xi}=d \bar{\xi}_{r} d \bar{\xi}_{\theta} d \bar{\xi}_{z}$. The explicit forms of $K$ for BCs employed in the present study are given below.

\section{Scattering kernel of the modified Maxwell-type condition}

$$
\text { For } i=I \text { or } O \text {, }
$$

$$
\begin{aligned}
K\left(\bar{\xi}, \xi ; \alpha_{i}, \beta_{i}, T_{i}, V_{i}\right) & \\
= & {\left[\left(1-\alpha_{i}\right) \delta\left(\xi_{r}+\bar{\xi}_{r}\right)+\alpha_{i} \frac{\left|\xi_{r}\right|}{R T_{i}} \exp \left(-\frac{\xi_{r}^{2}}{2 R T_{i}}\right)\right] } \\
& \times\left[\left(1-\beta_{i}\right) \delta\left(\xi_{\theta}-\bar{\xi}_{\theta}\right) \delta\left(\xi_{z}-\bar{\xi}_{z}\right)\right. \\
& \left.+\beta_{i} \frac{1}{2 \pi R T_{i}} \exp \left(-\frac{\left(\xi_{\theta}-V_{i}\right)^{2}+\xi_{z}^{2}}{2 R T_{i}}\right)\right],
\end{aligned}
$$

where $\delta$ is the Dirac delta function and $V_{O}=0$. The coefficients $\alpha_{I}$ and $\beta_{I}$ (or $\alpha_{O}$ and $\beta_{O}$ ) of the inner (or outer) cylinder have the following physical meanings: $\alpha_{i} \in[0,1]$ is the energy accommodation coefficient (EAC) associated with the normal velocity component and $\beta_{i} \in[0,1]$ is the TMAC. In terms of the notation introduced in Appendix A, where an accommodation coefficient $a_{c}(\psi)$ for an arbitrary function $\psi(\xi)$ is defined, those are written as $\alpha_{i}=a_{c}\left(\xi_{r}^{2}\right)$ and $\beta_{i}=a_{c}\left(\xi_{\theta}\right)=a_{c}\left(\xi_{z}\right)$.

Incidentally, the EAC associated with the tangential velocity is also given by $\beta_{i}$ (more precisely, $a_{c}\left[\left(\xi_{\theta}-V_{i}\right)^{2}\right]=$ $a_{c}\left(\xi_{z}^{2}\right)=\beta_{i}$; see Appendix A for a definition of $\left.a_{c}(\cdot)\right)$. In Appendix B, we show the expression of the above kernel (5) as a convex combination of the scattering kernel for the CL condition [Eq. (6) below] and an extension to $\beta_{i} \in(1,2]$.

\section{Scattering kernel of the CL condition}

For $i=I$ or $O$,

$$
\begin{aligned}
K\left(\bar{\xi}, \xi ; \alpha_{i}, \beta_{i}, T_{i}, V_{i}\right)= & K_{n}\left(\bar{\xi}_{r}, \xi_{r} ; \alpha_{i}, T_{i}\right) \\
& \times K_{t}\left(\bar{\xi}_{\theta}-V_{i}, \xi_{\theta}-V_{i} ; \beta_{i}, T_{i}\right) \\
& \times K_{t}\left(\bar{\xi}_{z}, \xi_{z} ; \beta_{i}, T_{i}\right),
\end{aligned}
$$

where

$$
\begin{aligned}
K_{n}(\bar{c}, c ; \alpha, T)= & \frac{|c|}{\alpha R T} I_{0}\left(\frac{(1-\alpha)^{1 / 2}}{\alpha R T} \bar{c} c\right) \\
& \times \exp \left(-\frac{c^{2}+(1-\alpha) \bar{c}^{2}}{2 \alpha R T}\right), \\
K_{t}(\bar{c}, c ; \beta, T)= & \frac{1}{[2 \pi \beta(2-\beta) R T]^{1 / 2}} \\
& \times \exp \left(-\frac{[c-(1-\beta) \bar{c}]^{2}}{2 \beta(2-\beta) R T}\right), \\
I_{0}(y)= & \frac{1}{2 \pi} \int_{0}^{2 \pi} \exp (y \cos \phi) d \phi .
\end{aligned}
$$

Here $I_{0}$ is the modified Bessel function of the first kind and zeroth order. The coefficient $\alpha_{i} \in[0,1]$ in Eq. (6) has the same physical meaning as in Eq. $(5) ; \beta_{i} \in[0,2]$ is again the TMAC, whereas the EAC associated with the tangential velocity is given by $\beta_{i}\left(2-\beta_{i}\right)$ in the case of the CL condition (i.e., $a_{c}\left[\left(\xi_{\theta}-V_{i}\right)^{2}\right]=a_{c}\left(\xi_{z}^{2}\right)=\beta_{i}\left(2-\beta_{i}\right)$; see Appendix A for a definition of $\left.a_{c}(\cdot)\right)$. When $\beta_{i}>1$, more than half of the impinging molecules experience backscattering (i.e., the scattering where the change in the direction of tangential velocity is larger than $\pi / 2$ ).

\section{Dimensionless parameters}

Rewriting the Boltzmann equation (2) and the BC (4) [with the scattering kernel (5) or (6)] in an appropriate dimensionless form, we see that the present problem is characterized by the following dimensionless parameters:

$$
\begin{aligned}
& r_{O} / r_{I}, \quad T_{O} / T_{I}, \quad U_{I}\left[\equiv V_{I} /\left(2 R T_{I}\right)^{1 / 2}\right], \\
& \alpha_{I}, \quad \beta_{I}, \quad \alpha_{O}, \quad \beta_{O}, \quad \mathrm{Kn}\left(\equiv \ell_{I} / r_{I}\right) .
\end{aligned}
$$

The Knudsen number Kn, which indicates the degree of rarefaction, is defined as the ratio of the mean free path $\ell_{I}$ of molecules in an equilibrium state at rest with density $\rho_{\mathrm{av}}$ and temperature $T_{I}$ to the radius $r_{I}$ of the inner cylinder. 
Here $\rho_{\mathrm{av}}$ denotes the average density of the gas between the cylinders; $\ell_{I}=m /\left(2^{1 / 2} \pi d^{2} \rho_{\mathrm{av}}\right)$ in the case of the hard-sphere (HS) molecular gas ( $m$ and $d$ are the molecular mass and diameter) and $\ell_{I}=\left(8 R T_{I} / \pi\right)^{1 / 2} /\left(A \rho_{\mathrm{av}}\right)$ in the case of the BGK model.

\section{ASYMPTOTIC ANALYSIS FOR SMALL KNUDSEN NUMBERS}

In this section, we carry out an asymptotic analysis of the boundary-value problem (2) and (4) for small Knudsen numbers, in the case where the TMAC $\beta_{i}$ is also small [the actual analysis is performed for dimensionless versions of Eqs. (2) and (4)]. More precisely, we consider the following two cases:

Case 1: $\alpha_{i}=\hat{\alpha}_{i} \epsilon, \beta_{i}=\hat{\beta}_{i} \epsilon$,

Case 2: $\alpha_{i}=O(1), \quad \beta_{i}=\hat{\beta}_{i} \epsilon$, where $\epsilon \equiv\left(\pi^{1 / 2} / 2\right) \mathrm{Kn}$. Since the procedure of the analysis is essentially the same as in Ref. [3] (see also Refs. [21,22]), we show its outline only (notice the difference in the definitions of $\alpha_{i}$ and $\beta_{i}$ here and those in Ref. [3]).

\section{A. Fluid-dynamic equation}

Let $\epsilon$ be small. First, putting aside the boundary condition, we seek a solution of the Boltzmann equation [dimensionless version of Eq. (2)] varying moderately in space in a power series of $\epsilon$. This solution is called the Hilbert solution. According to Ref. [3], the Hilbert solution at the leading order (the order of $\epsilon^{0}$ ) is a local Maxwellian. From the solvability conditions for the solution at the order of $\epsilon^{1}$ and $\epsilon^{2}$, the following fluid-dynamic set of equations for the macroscopic quantities at the leading order is derived:

$$
\begin{aligned}
\frac{d \hat{p}_{0}}{d \hat{r}}-\frac{2 \hat{\rho}_{0} u_{\theta 0}^{2}}{\hat{r}} & =0, \quad(8) \\
\frac{d}{d \hat{r}}\left[\gamma_{1} \hat{r}^{2} \hat{T}_{0}^{1 / 2}\left(\frac{d u_{\theta 0}}{d \hat{r}}-\frac{u_{\theta 0}}{\hat{r}}\right)\right] & =0, \quad(8) \\
\frac{5}{4} \frac{d}{d \hat{r}}\left(\gamma_{2} \hat{r} \hat{T}_{0}^{1 / 2} \frac{d \hat{T}_{0}}{d \hat{r}}\right)+\gamma_{1} \hat{r} \hat{T}_{0}^{1 / 2}\left(\frac{d u_{\theta 0}}{d \hat{r}}-\frac{u_{\theta 0}}{\hat{r}}\right)^{2} & =0, \quad(8 \\
\hat{p}_{0} & =\hat{\rho}_{0} \hat{T}_{0},
\end{aligned}
$$

where $\hat{r}=r / r_{I}$. The dependent variables in Eq. (8) are the dimensionless macroscopic quantities defined as $\hat{\rho}=\rho / \rho_{\mathrm{av}}$, $u_{\theta}=v_{\theta} /\left(2 R T_{I}\right)^{1 / 2}, \hat{T}=T / T_{I}$, and $\hat{p}=p /\left(R \rho_{\mathrm{av}} T_{I}\right)$ at the order of $\epsilon^{0}$ : i.e., the leading term in the power series,

$$
h=h_{0}+h_{1} \epsilon+h_{2} \epsilon^{2}+\cdots \quad\left(h=\hat{\rho}, u_{\theta}, \hat{T}, \hat{p}\right) .
$$

$\gamma_{1}$ and $\gamma_{2}$ are functions of $\hat{T}_{0}$, whose functional forms are determined by the intermolecular force: $\gamma_{1}=1.270042$ and $\gamma_{2}=1.922284$ in the case of the HS molecules and $\gamma_{1}=\gamma_{2}=$ $\hat{T}_{0}^{1 / 2}$ in the case of the BGK model equation. Since $\gamma_{1} \hat{T}_{0}^{1 / 2}$ and $\gamma_{2} \hat{T}_{0}^{1 / 2}$ correspond, respectively, to the dimensionless viscosity and thermal conductivity, the above set of equations (8) is equivalent to the compressible Navier-Stokes set. We should note again that the above set (8) has been derived in Ref. [3]; the explicit form of the Hilbert solution up to $O\left(\epsilon^{1}\right)$ is shown in the same reference.

\section{B. Boundary conditions for the fluid-dynamic equation}

Next we take into account the kinetic boundary condition (KBC) [dimensionless version of Eq. (4)]. Let us first consider the case of $\beta_{i}=O(1)$. In this case, the Hilbert solution at the leading order (i.e., a local Maxwellian) will satisfy the KBC if the boundary values of $u_{\theta 0}$ and $\hat{T}_{0}$ coincide with the velocity and temperature of the cylinders. That is, as a $\mathrm{BC}$ for the fluid-dynamic set (8), the nonslip condition is required:

$$
U_{i}-u_{\theta 0}=0, \quad \hat{T}_{w i}-\hat{T}_{0}=0 \quad \text { at } \quad \hat{r}=\hat{r}_{i},
$$

where $i=I$ (or $i=O$ ) on the inner (or outer) cylinder, $\hat{r}_{I}=1$, $\hat{r}_{O}=r_{O} / r_{I}, U_{O}=0, \hat{T}_{w I}=1$, and $\hat{T}_{w O}=T_{O} / T_{I}$.

In the case of $\beta_{i}=O\left(\epsilon^{1}\right)$ [and $\alpha_{i}=O\left(\epsilon^{1}\right)$ ], however, the $\mathrm{KBC}$ at the leading order puts no restrictions on the boundary values of $u_{\theta 0}$ (and $\hat{T}_{0}$ ). To determine those boundary values, we need to proceed to the next order.

\section{Case 1}

In this case, the $\mathrm{KBC}$ at the leading order reduces to the specular reflection, which is satisfied unconditionally. At the next order, however, the Hilbert solution cannot satisfy the KBC. Thus we need to introduce a correction (called the Knudsen-layer correction) in a thin layer [with a thickness of $O\left(\ell_{I}\right)$ ] adjacent to the boundary. The total solution (Hilbert solution + Knudsen-layer correction) will solve the original boundary-value problem at $O\left(\epsilon^{1}\right)$.

As in Ref. [3], the problem for the Knudsen layer is formulated as a half-space boundary-value problem of the linearized Boltzmann equation, which in general can be solved only numerically. By solving it, one obtains the Knudsen layer at $O\left(\epsilon^{1}\right)$ and, at the same time, the BCs for $u_{\theta 0}$ and $\hat{T}_{0}$. In the present study, however, we need the latter BCs only. Thus we follow the procedure in Refs. [23-25], by which one can derive the latter only without solving the Knudsen-layer problem explicitly. The result for the modified Maxwell-type condition [Eq. (4) with (5)] is given as follows: at $\hat{r}=\hat{r}_{i}$,

$$
\begin{aligned}
& \pi^{1 / 2} \gamma_{1}\left(\frac{d u_{\theta 0}}{d \hat{r}}-\frac{u_{\theta 0}}{\hat{r}_{i}}\right)+\hat{\beta}_{i} \hat{\rho}_{0}\left(U_{i}-u_{\theta 0}\right) n_{i}=0 \\
& \frac{5}{4} \pi^{1 / 2} \gamma_{2} \frac{d \hat{T}_{0}}{d \hat{r}}+\frac{\hat{\alpha}_{i}+\hat{\beta}_{i}}{2} \hat{\rho}_{0}\left(\hat{T}_{w i}-\hat{T}_{0}\right) n_{i} \\
& \quad+\frac{1}{2} \hat{\beta}_{i} \hat{\rho}_{0}\left(U_{i}-u_{\theta 0}\right)^{2} n_{i}=0 .
\end{aligned}
$$

Note that Eq. (10) with $\hat{\alpha}_{i}=\hat{\beta}_{i}$ is essentially the same as the corresponding result in Ref. [3] derived for the (standard) Maxwell-type condition. In the case of the CL condition [Eq. (4) with (6)], one obtains Eq. (10a) and

$$
\frac{5}{4} \pi^{1 / 2} \gamma_{2} \frac{d \hat{T}_{0}}{d \hat{r}}+\frac{\hat{\alpha}_{i}+2 \hat{\beta}_{i}}{2} \hat{\rho}_{0}\left(\hat{T}_{w i}-\hat{T}_{0}\right) n_{i}=0,
$$

instead of Eq. (10b). The above BCs (10) and (11) approach the nonslip BC (9) as $\hat{\beta}_{i} \rightarrow \infty$. 


\section{Case 2}

In this case, the $\mathrm{KBC}$ at the leading order reduces to the specular reflection with respect to the tangential velocity only [corresponding to the limit $\beta_{i} \rightarrow 0$ of Eq. (5) or (6)]. The Hilbert solution at the leading order (a local Maxwellian) can satisfy this condition if the boundary value of $\hat{T}_{0}$ coincides with temperature of the cylinder. That is, the nonslip condition for $\hat{T}_{0}$ is required. To determine the boundary value of $u_{\theta 0}$, we again consider the Knudsen layer at $O\left(\epsilon^{1}\right)$ and follow the same procedure as before. Finally, we obtain the following BCs for $u_{\theta 0}$ and $\hat{T}_{0}:$ at $\hat{r}=\hat{r}_{i}$,

$$
\begin{array}{r}
\pi^{1 / 2} \gamma_{1}\left(\frac{d u_{\theta 0}}{d \hat{r}}-\frac{u_{\theta 0}}{\hat{r}_{i}}\right)+\hat{\beta}_{i} \hat{\rho}_{0}\left(U_{i}-u_{\theta 0}\right) n_{i}=0, \\
\hat{T}_{w i}-\hat{T}_{0}=0
\end{array}
$$

for both the modified Maxwell-type and CL conditions. The above $\mathrm{BC}$ (12) is derived also as the limit $\hat{\alpha}_{i} \rightarrow \infty$ of the $\mathrm{BC}$ (10) [or (11)] in Case 1.

\section{Numerical solutions of the fluid-dynamic equation}

Numerical solutions of the fluid-dynamic equation (8) for the HS molecules with the BC (10), (11), or (12) are shown below. Incidentally, Eq. (8) for the BGK model (with $\gamma_{1}=$ $\gamma_{2}=\hat{T}_{0}^{1 / 2}$ ) gives similar results (see Fig. 9 appearing later). For simplicity, we assume that both cylinders have common accommodation coefficients, i.e., $\hat{\alpha}_{I}=\hat{\alpha}_{O}(\equiv \hat{\alpha})$ and $\hat{\beta}_{I}=\hat{\beta}_{O}$ $(\equiv \hat{\beta})$.

Figure 1 shows results with the $\mathrm{BC}$ (10) (corresponding to the modified Maxwell-type condition) or (12) (to both KBCs) in the case of $r_{O} / r_{I}=2, T_{O} / T_{I}=1$, and $U_{I}=0.5$. The profile of the velocity $u_{\theta 0}$ is inverted for $\hat{\beta} \lesssim 0.5$ in this case. Since $\hat{\alpha}$ appears only in the BC (10b) for the temperature, the velocity profile is almost independent of $\hat{\alpha}$. Figure 2 shows results with the $\mathrm{BC}(10 \mathrm{a})$ and (11) (corresponding to the CL condition) for the same case as in Fig. 1(a). Comparing Figs. 1(a) and 2, we see that the velocity profiles for both KBCs are almost identical.

Figures 3 and 4 show the dependence of the velocity profile on $U_{I}$ and $r_{O} / r_{I}$, respectively. As in Figs. 1 and 2, the inverted velocity profile occurs for sufficiently small $\hat{\beta}$. The velocity slips (or $\left|U_{i}-u_{\theta 0}\right|$ ) on both cylinders increase with decreasing $\hat{\beta}$, whereas the slip on the inner cylinder grows faster, resulting in the inverted profile. This is because the torque exerted on the gas by the cylinder is proportional to $\hat{r}_{i}^{2}\left|U_{i}-u_{\theta 0}\right|$. To be more specific, let us denote the magnitude of the torque (per unit length in $z$ direction) as $2 \pi r_{I}^{2}\left(R \rho_{\mathrm{av}} T_{I}\right) \hat{N}_{i}$, where $i=I$ (or $i=O$ ) for the inner (or outer) cylinder. Then $\hat{N}_{i}$ up to $O(\epsilon)$ is given as

$$
\hat{N}_{i}=\pi^{-1 / 2} \hat{r}_{i}^{2} \hat{\beta}_{i} \hat{\rho}_{0}\left(\hat{r}_{i}\right) \hat{T}_{0}^{1 / 2}\left(\hat{r}_{i}\right)\left|U_{i}-u_{\theta 0}\left(\hat{r}_{i}\right)\right| \epsilon .
$$

If we ignore the differences between $\hat{\rho}_{0}(1) \hat{T}_{0}^{1 / 2}(1)$ and $\hat{\rho}_{0}\left(\hat{r}_{O}\right) \hat{T}_{0}^{1 / 2}\left(\hat{r}_{O}\right)$ (they are less than $3 \%$ when $\hat{\beta}<2$ in Figs. 1-4), we deduce from $\hat{N}_{O}=\hat{N}_{I}$ that $u_{\theta 0}\left(\hat{r}_{O}\right) \geqslant u_{\theta 0}(1)$ when

$$
u_{\theta 0}\left(\hat{r}_{O}\right) \geqslant \frac{U_{I}}{1+\hat{r}_{O}^{2}\left(\hat{\beta}_{O} / \hat{\beta}_{I}\right)} .
$$
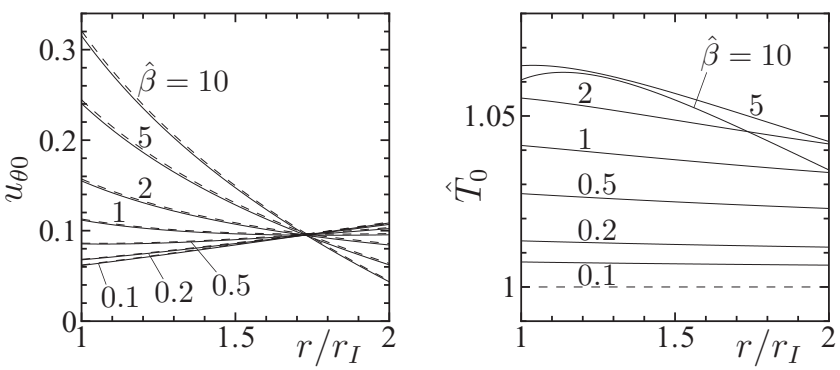

(a) $\hat{\alpha}=1$
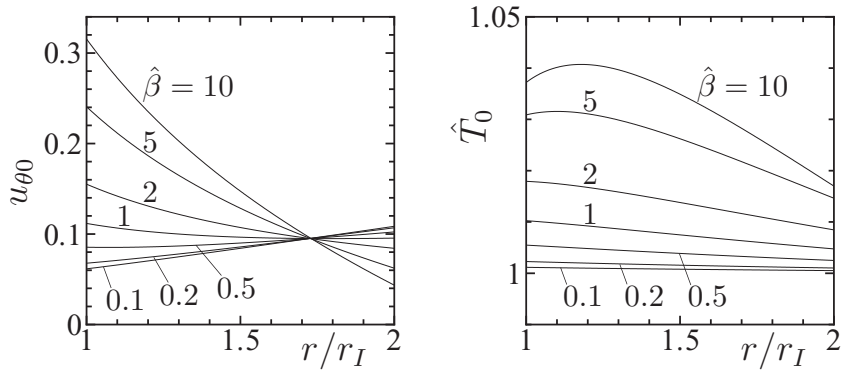

(b) $\hat{\alpha}=10$
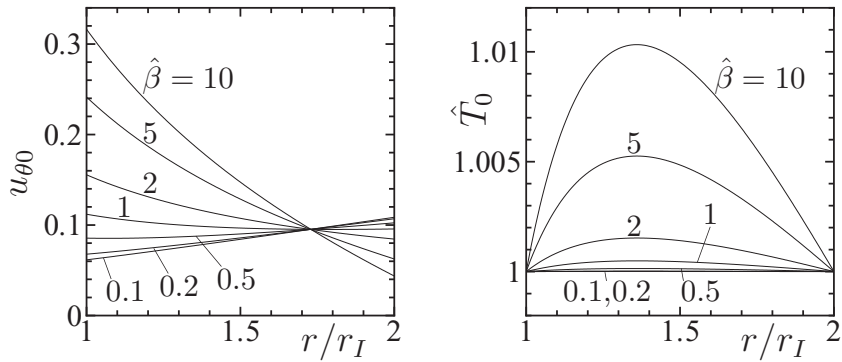

(c) $\hat{\alpha} \rightarrow \infty$

FIG. 1. Solutions $u_{\theta 0}$ and $\hat{T}_{0}$ of the fluid-dynamic equation (8) (for the HS molecules) with the boundary condition (10) [in panel (a) and (b)] or (12) [in panel (c)] for $r_{O} / r_{I}=2, T_{O} / T_{I}=1, U_{I}=0.5$, and various values of $\hat{\alpha}\left(\equiv \hat{\alpha}_{I}=\hat{\alpha}_{O}\right)$ and $\hat{\beta}\left(\equiv \hat{\beta}_{I}=\hat{\beta}_{O}\right)$. The dashed lines in panel (a) indicate the corresponding results of the linearized set (15) with (16)

This inequality gives a rough estimate for the velocity slip on the outer cylinder when the inverted profile arises, which is actually satisfied in Figs. 1-4. The above inequality can naturally be derived also from the exact solution of the
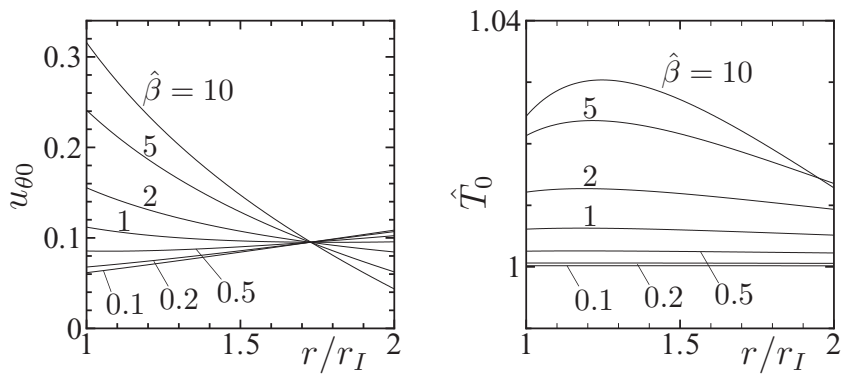

FIG. 2. Solutions $u_{\theta 0}$ and $\hat{T}_{0}$ of the fluid-dynamic equation (8) (for the HS molecules) with the boundary conditions (10a) and (11) for $r_{O} / r_{I}=2, U_{I}=0.5, T_{O} / T_{I}=1$, and $\hat{\alpha}=1$. 


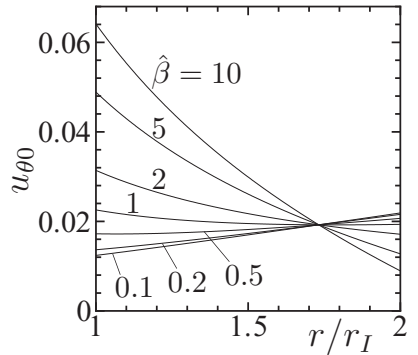

(a) $U_{I}=0.1$

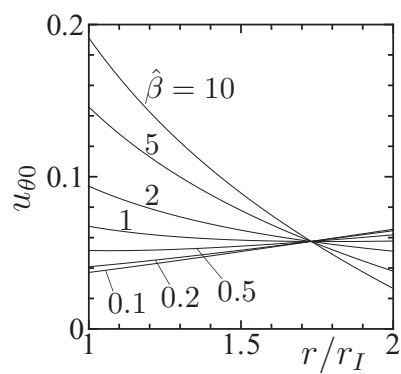

(b) $U_{I}=0.3$
FIG. 3. Solutions $u_{\theta 0}$ of the fluid-dynamic equation (8) (for the HS molecules) with the boundary condition (10) for $r_{O} / r_{I}=2$, $T_{O} / T_{I}=1$, and $\hat{\alpha}=1$. (a) $U_{I}=0.1$ and (b) $U_{I}=0.3$.

linearized equation shown in the next section (see also Appendix C).

\section{Linearization}

We next linearize the fluid-dynamic equation (8) and the BCs (10), (11), and (12) under the assumption that $\left|U_{I}\right| \ll 1$ and $\left|\hat{T}_{w O}-1\right| \ll 1$ (but $\left|U_{I}\right| \gg \mathrm{Kn}$ ). If we put $\hat{\rho}_{0}=1+\omega$, $u_{\theta 0}=u, \hat{T}_{0}=1+\tau$, and $\hat{p}_{0}=1+P$ with $|\omega| \ll 1,|u| \ll 1$, $|\tau| \ll 1$, and $|P| \ll 1$, and neglect the higher-order terms, then the fluid-dynamic equation $(8)$ reduces to

$$
\begin{aligned}
\frac{d P}{d \hat{r}} & =0, \quad \frac{d}{d \hat{r}}\left[\hat{r}^{2}\left(\frac{d u}{d \hat{r}}-\frac{u}{\hat{r}}\right)\right]=0, \\
\frac{d}{d \hat{r}}\left(\hat{r} \frac{d \tau}{d \hat{r}}\right) & =0, \quad P=\omega+\tau .
\end{aligned}
$$

The BCs (10) and (11) in Case 1 are rewritten as follows: at $\hat{r}=\hat{r}_{i}$,

$$
\begin{aligned}
& \pi^{1 / 2} \gamma_{1}^{*}\left(\frac{d u}{d \hat{r}}-\frac{u}{\hat{r}_{i}}\right)+\hat{\beta}_{i}\left(U_{i}-u\right) n_{i}=0, \\
& \frac{5}{4} \pi^{1 / 2} \gamma_{2}^{*} \frac{d \tau}{d \hat{r}}+\frac{\hat{\alpha}_{i}+c \hat{\beta}_{i}}{2}\left(\tau_{w i}-\tau\right) n_{i}=0,
\end{aligned}
$$

where $c=1$ for the modified Maxwell-type condition, $c=2$ for the CL condition, $\tau_{w I}=0, \tau_{w O}=\hat{T}_{w O}-1$, and $\gamma_{1}^{*}$ and $\gamma_{2}^{*}$ denote, respectively, $\gamma_{1}$ and $\gamma_{2}$ at $\hat{T}_{0}=1$. The BC in Case 2 is

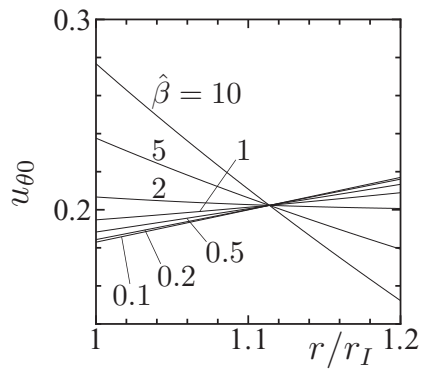

(a) $r_{O} / r_{I}=1.2$

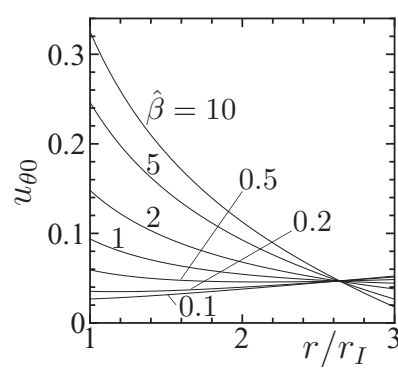

(b) $r_{O} / r_{I}=3$
FIG. 4. Solutions $u_{\theta 0}$ of the fluid-dynamic equation (8) (for the HS molecules) with the boundary condition (10) for $T_{O} / T_{I}=1$, $U_{I}=0.5$, and $\hat{\alpha}=1$. (a) $r_{O} / r_{I}=1.2$ and (b) $r_{O} / r_{I}=3$.

given by Eq. (16a) and $\tau_{w i}-\tau=0$ instead of Eq. (16b). Note again that Eq. (16) with $c=1$ and $\hat{\alpha}_{i}=\hat{\beta}_{i}$ is essentially the same as the corresponding result in Ref. [3] for the (standard) Maxwell-type condition.

The flow velocity $u$ is decoupled from the temperature $\tau$ in the above linearized system (15) and (16). Thus $u$ is independent of $\hat{\alpha}_{i}$ but depends only on $\hat{\beta}_{i}$ corresponding to the TMAC. The above system can be solved analytically and gives the velocity field $u$ corresponding to the solution of the incompressible Navier-Stokes equation. As indicated by dashed lines in Fig. 1(a), the solution $u$ does not differ much from the result of the compressible Navier-Stokes set even when $U_{I}=0.5$. In Appendix $\mathrm{C}$, the explicit form of the analytical solution is shown and two kinds of criterion for the occurrence of the velocity inversion, one of which will be compared with numerical results of the BGK model in Fig. 10 later, are derived from it.

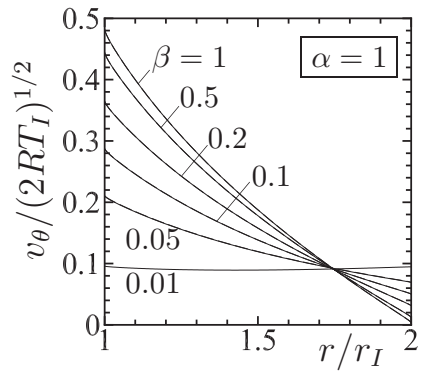

(a) $\mathrm{Kn}=0.02$

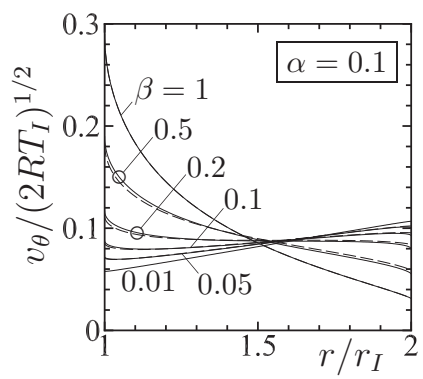

(c) $\mathrm{Kn}=1$
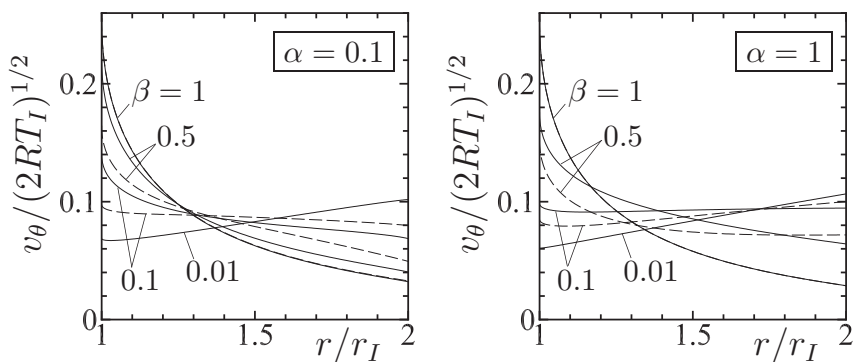

(d) $\mathrm{Kn}=10$

FIG. 5. Profiles of the flow velocity $v_{\theta}$ for the BGK model equation in the case of $r_{O} / r_{I}=2, U_{I}=0.5$, and $T_{O} / T_{I}=1$. (a) $\mathrm{Kn}=0.02$, (b) $\mathrm{Kn}=0.1$, (c) $\mathrm{Kn}=1$, and (d) $\mathrm{Kn}=10$. The solid lines indicate the results of the modified Maxwell-type BC, and the dashed lines (for $\beta \geqslant 0.05$ ) those of the Cercignani-Lampis BC. 


\section{NUMERICAL ANALYSIS FOR A WIDE RANGE OF THE KNUDSEN NUMBER}

A numerical analysis of the BGK model equation [i.e., Eq. (2) with (3)] via a finite-difference method is carried out to investigate the behavior of the gas for a wide range of the Knudsen number. Essentially the same finite-difference scheme as that devised in Ref. [26] is employed, where the discontinuity of the VDF appearing in a gas around a convex body [27] is correctly taken into account.

In this section, we restrict ourselves to the case of $r_{O} / r_{I}=2$ as well as $\alpha_{I}=\alpha_{O}(\equiv \alpha)$ and $\beta_{I}=\beta_{O}(\equiv \beta)$ as in Sec. IIIC. The computations are performed mainly for the modified Maxwell-type condition [Eq. (4) with (5)], whereas some auxiliary computations for the CL condition [Eq. (4) with (6)] are also performed for comparison; this is because the former condition is easier to compute in the case of a finite-difference method. Some data for the computation are given in Appendix D.
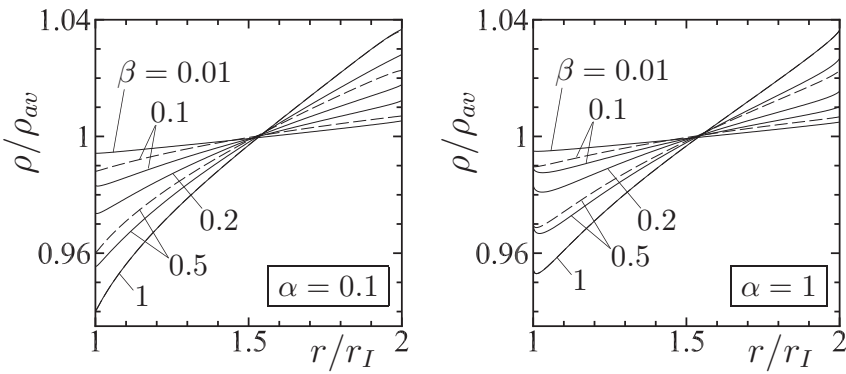

(a) $\mathrm{Kn}=0.1$
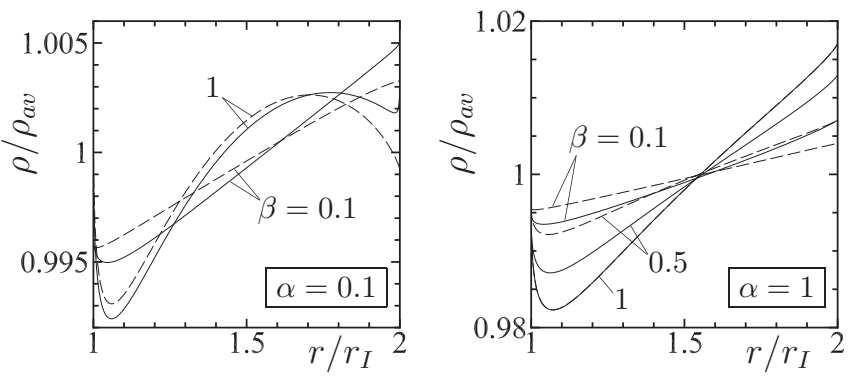

(b) $\mathrm{Kn}=1$
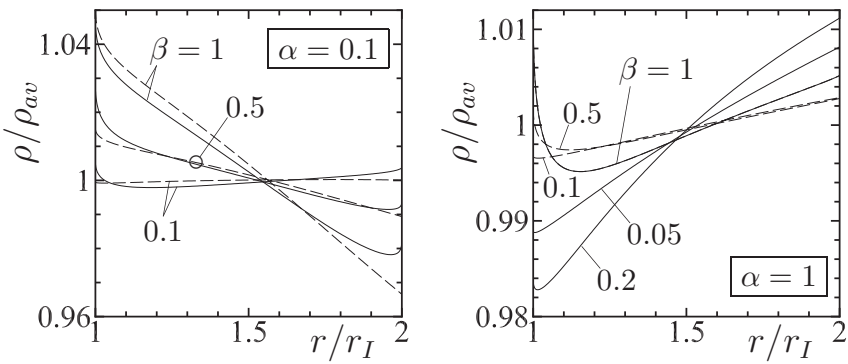

(c) $\mathrm{Kn}=10$

FIG. 6. Profiles of the density $\rho$ for the BGK model equation in the case of $r_{O} / r_{I}=2, U_{I}=0.5$, and $T_{O} / T_{I}=1$. (a) $\mathrm{Kn}=0.1$, (b) $\mathrm{Kn}=1$, and (c) $\mathrm{Kn}=10$. The solid lines indicate the results of the modified Maxwell-type BC, and the dashed lines (for $\beta=0.1,0.5$, and 1) those of the Cercignani-Lampis BC.
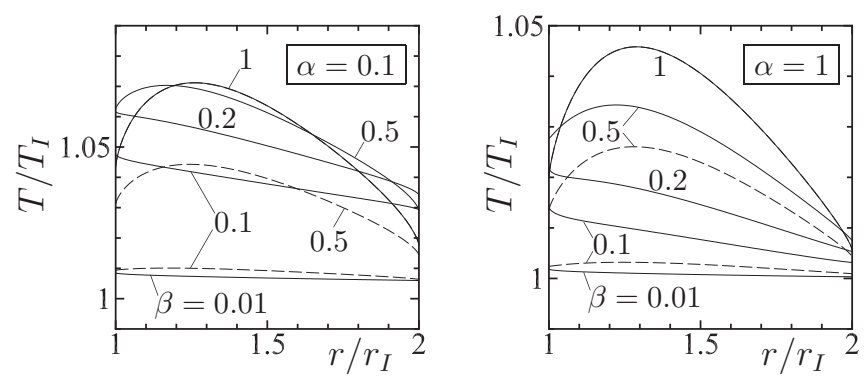

(a) $\mathrm{Kn}=0.1$
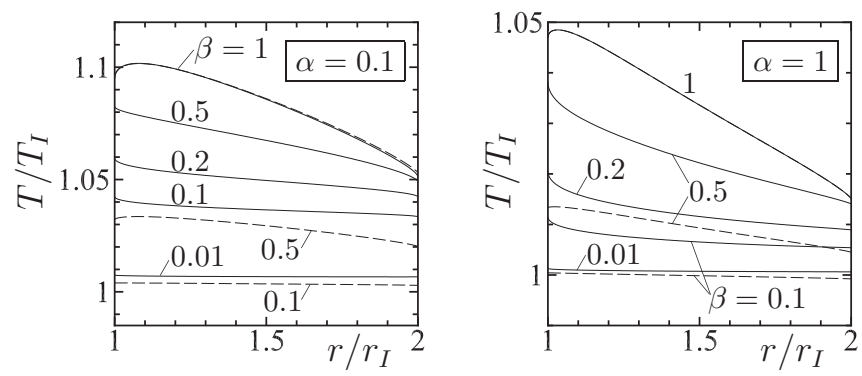

(b) $\mathrm{Kn}=1$
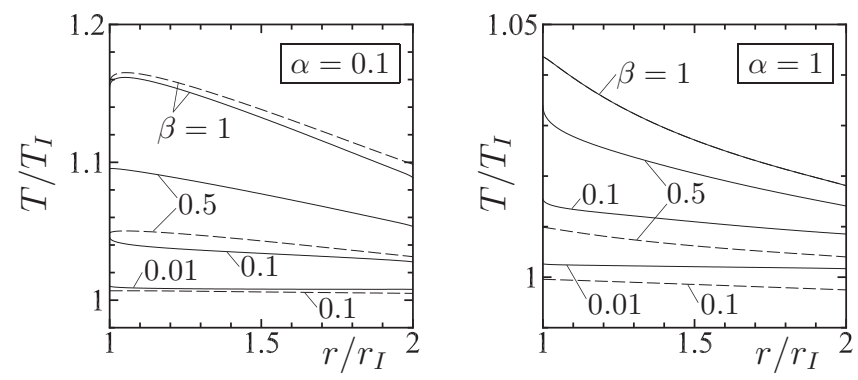

(c) $\mathrm{Kn}=10$

FIG. 7. Profiles of the temperature $T$ for the BGK model equation in the case of $r_{O} / r_{I}=2, U_{I}=0.5$, and $T_{O} / T_{I}=1$. (a) $\mathrm{Kn}=0.1$, (b) $\mathrm{Kn}=1$, and (c) $\mathrm{Kn}=10$. The solid lines indicate the results of the modified Maxwell-type $\mathrm{BC}$, and the dashed lines (for $\beta=0.1$, 0.5 , and 1) those of the Cercignani-Lampis BC.

\section{A. Profiles of the macroscopic quantities}

Figures 5-7 show profiles of the flow velocity $v_{\theta}$, density $\rho$, and temperature $T$ for various values of $\alpha, \beta$, and $\mathrm{Kn}$ in the case of $r_{O} / r_{I}=2, U_{I}=0.5$, and $T_{O} / T_{I}=1$. Note that when $\alpha=\beta=1$, both the modified Maxwell-type BC and the CL condition reduce to the diffuse reflection condition and give exactly the same profiles.

The profile of $v_{\theta}$ for small $\mathrm{Kn}$ is almost independent of $\alpha$ and practically depends on $\beta$ (or the TMAC) only. Thus only the results for $\alpha=1$ are shown in Figs. 5(a) and 5(b). The results of the modified Maxwell-type condition and those of the CL condition with the same $\beta$ are indistinguishable in Figs. 5(a) and 5(b), since the TMAC is the same for both BCs. As $\mathrm{Kn}$ increases, however, the dependence of $v_{\theta}$ on $\alpha$ as well as on the type of BC appears [Figs. 5(c) and 5(d)], since the gas behavior becomes sensitive to details of $\mathrm{BC}$ (details of the velocity distribution of molecules reflected on the boundary). 


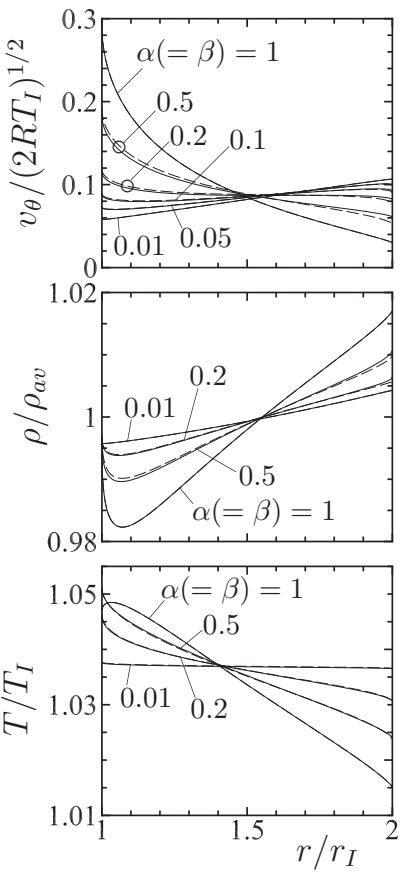

(a) $\mathrm{Kn}=1$
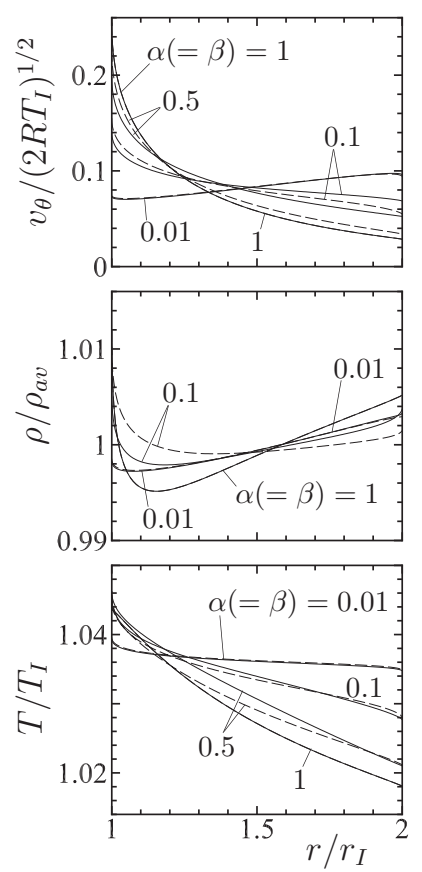

(b) $\mathrm{Kn}=10$
FIG. 8. Comparison of results for the BGK model equation with the modified Maxwell-type $\mathrm{BC}$ (with $\alpha=\beta$ ) and those with the standard Maxwell-type BC (with the same accommodation coefficient) in the case of $r_{O} / r_{I}=2, U_{I}=0.5$, and $T_{O} / T_{I}=1$. (a) $\mathrm{Kn}=1$ and (b) $\mathrm{Kn}=10$. The solid lines (or the dashed lines) indicate results with the modified (or standard) Maxwell-type BC.

On the other hand, as seen in Figs. 6 and 7, the profiles of $\rho$ and $T$ depend on both $\alpha$ and $\beta$. Those for the modified Maxwell-type condition do not agree well with those for the CL condition with the same $\alpha$ and $\beta$ (except when $\alpha=\beta=1$ ), even when $\mathrm{Kn}$ is small. This is because the EAC is different; as mentioned in Sec. II C, $a_{c}\left[\left(\xi_{\theta}-V_{i}\right)^{2}\right]=\beta$ for the modified Maxwell-type condition, whereas $a_{c}\left[\left(\xi_{\theta}-V_{i}\right)^{2}\right]=\beta(2-\beta)$ for the $\mathrm{CL}$ condition $\left[a_{c}\left(\xi_{r}^{2}\right)=\alpha\right.$ for both BCs].

Figure 8 shows a comparison of the results of the modified Maxwell-type condition with $\alpha=\beta$ and those of the (standard) Maxwell-type condition with the same accommodation coefficient. In this case, both BCs have common TMAC and EAC. Thus all the profiles of $v_{\theta}, \rho$, and $T$ show good agreement when $\mathrm{Kn} \lesssim 1$. Naturally, the disagreement appears as $\mathrm{Kn}$ increases, since the profiles become sensitive to details of $\mathrm{BC}$.

The profiles of the flow velocity for small Kn in Figs. 5(a) and 5(b) and those of the temperature in Fig. 7(a) are similar to the results of the asymptotic analysis shown in Fig. 1. More direct comparisons when $\epsilon\left[=\left(\pi^{1 / 2} / 2\right) \mathrm{Kn}\right]=0.02$ are shown in Fig. 9. The results of the BGK model are well reproduced by the fluid-dynamic set (8) with the $\mathrm{BC}(10)$ even when $\beta=1$ and/or $\alpha=1$. The results obtained with the nonslip BC (9), which correspond to the limit $\hat{\beta} \rightarrow \infty$, are also shown for comparison. As seen in panel (b), the temperature profiles obtained with the BC (12) (corresponding to the limit $\hat{\alpha} \rightarrow$ $\infty$ ) are less accurate, whereas they are consistent because the differences from the results of the BGK model are smaller than $\epsilon$.

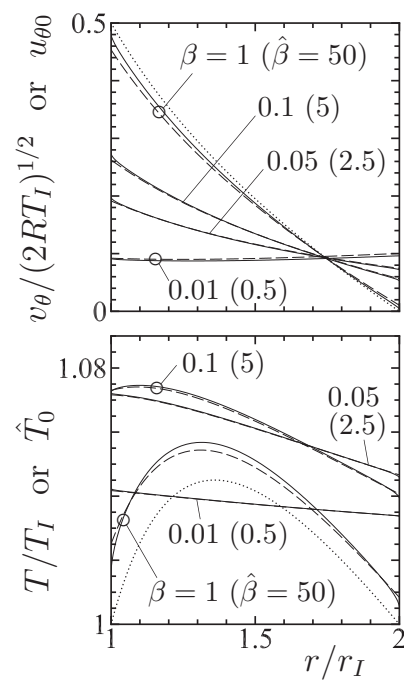

(a) $\alpha=0.01(\hat{\alpha}=0.5)$

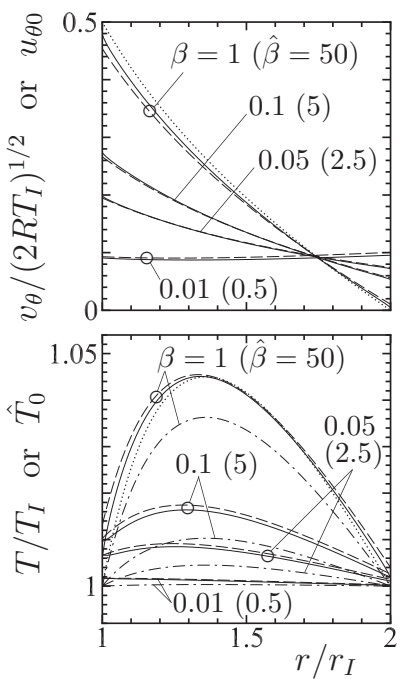

(b) $\alpha=1(\hat{\alpha}=50)$
FIG. 9. Comparison of the flow-velocity and temperature profiles for the BGK model equation with the modified Maxwell-type BC and the corresponding $u_{\theta 0}$ and $\hat{T}_{0}$ of the fluid-dynamic equation (8) (with $\left.\gamma_{1}=\gamma_{2}=\hat{T}_{0}^{1 / 2}\right)$ in the case of $r_{O} / r_{I}=2, U_{I}=0.5, T_{O} / T_{I}=1$, and $\epsilon\left[=\left(\pi^{1 / 2} / 2\right) \mathrm{Kn}\right]=0.02$. The solid lines indicate results of the BGK model for $\alpha=0.01$ (or $\alpha=1$ ) and $\beta=0.01,0.05,0.1$, and 1 in panel (a) [or in panel (b)]. The dashed lines indicate results of the fluid-dynamic equation with the $\mathrm{BC}(10)$ for $\hat{\alpha}=0.5$ (or $\hat{\alpha}=50$ ) and $\hat{\beta}=0.5,2.5,5$, and 50 in panel (a) [or in panel (b)]; the dashed-dotted lines for $\hat{T}_{0}$ in panel (b) indicate results with the BC (12) for the same $\hat{\beta}$ 's; the dotted lines indicate results with the nonslip BC (9).

\section{B. Critical value of $\boldsymbol{\beta}$ for onset of the inverted velocity profile}

As seen in Fig. 5, the inversion of the flow velocity profile occurs when $\beta$ is sufficiently small. The critical value $\beta_{c}$ of $\beta$ for the onset of the inversion varies depending on $\mathrm{Kn}, \alpha$, and the other parameters. Figure 10 shows $\beta_{c}$ of the modified Maxwell-type BC as a function of Kn for several pairs of $\alpha$ and $T_{O} / T_{I}$. Here $\beta_{c}$ is defined to be the maximum value of $\beta$ when a positive gradient of $v_{\theta}$ appears in the gap between the cylinders [thus $v_{\theta}\left(r_{O}\right)>v_{\theta}\left(r_{I}\right)$ may not be achieved].

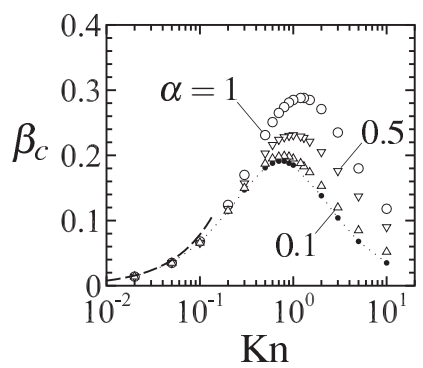

(a) $T_{O} / T_{I}=1$

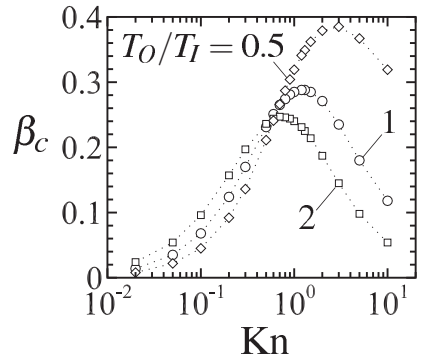

(b) $\alpha=1$
FIG. 10. Critical value $\beta_{c}$ vs $\mathrm{Kn}$ for the BGK model equation with the modified Maxwell-type BC in the case of $r_{O} / r_{I}=2$ and $U_{I}=0.5$. (a) $T_{O} / T_{I}=1$ and (b) $\alpha=1$. In panel (a), the filled circles indicate the critical values for the (standard) Maxwell-type $\mathrm{BC}$ reported in Ref. [3] and the dashed line those determined by Eq. (C3). 
The $\beta_{c}$ takes its maximum at an intermediate $\mathrm{Kn}$. When $\mathrm{Kn} \lesssim 1$, the velocity profile is insensitive to the type of $\mathrm{BC}$ as seen in Figs. 5(a), 5(b), and 8(a). Thus it is natural that, as seen in Fig. 10(a), $\beta_{c}$ converges to the critical value for the (standard) Maxwell-type BC as Kn decreases. In addition, $\beta_{c}$ for $T_{O} / T_{I}=1$ in panel (a) approaches the criterion (C3) as Kn decreases, which is derived from the solution of the linearized system (15) and (16) in Appendix C.

Finally, we should note that some of the previous experiments reported rather small values of accommodation coefficients for well-controlled clean surfaces; see Refs. [28,29] for the TMAC and Refs. [30,31] for the EAC. According to those references, the values of $\beta_{c}$ in Fig. 10 (for intermediate $\mathrm{Kn}$ ) are within the reach of experimental verification.

\section{CONCLUDING REMARKS}

In the present study, the cylindrical Couette flow of a rarefied gas between a rotating inner cylinder and a stationary outer cylinder was investigated on the basis of kinetic theory. Two kinds of kinetic boundary conditions, i.e., (i) the modified Maxwell-type condition and (ii) the CL condition, were employed on the surface of the cylinder, both of which can adjust the TMAC separately from another accommodation coefficient associated with the normal velocity component. By using those boundary conditions, we aimed to distinguish the effect of each accommodation coefficient on the behavior of the gas, especially on the profile of the circumferential flow velocity.

We carried out an asymptotic analysis of the Boltzmann equation for small Knudsen numbers, showed the fluiddynamic equation describing the behavior of the macroscopic quantities at the leading order, and derived the appropriate boundary conditions on the assumption that the TMAC is small (of the order of the Knudsen number). The numerical solutions of the fluid-dynamic system indicate that the flow velocity profile practically depends on the TMAC only. Further, when the rotation of the inner cylinder was slow, we linearized the above system and showed that the flow velocity is decoupled from the temperature field and thus exactly depends on the TMAC only.

Then we carried out the numerical analysis of the BGK model equation by means of a finite-difference method and obtained profiles of the macroscopic quantities for a wide range of the Knudsen number and the accommodation coefficients. The dependence of those macroscopic profiles on the accommodation coefficients and on the type of the boundary condition is clarified. We also made a comparison with the results of the asymptotic analysis and confirmed that the numerical solutions of the BGK model for small Kn were well reproduced by them. Finally, we obtained the critical values of TMAC for the onset of the inverted velocity profile in various cases. The results show that the critical value, as a function of the Knudsen number Kn, has a maximum at an intermediate $\mathrm{Kn}$ and converges to the previous result [3] for the (standard) Maxwell-type condition as Kn decreases.

\section{APPENDIX A: ACCOMMODATION COEFFICIENTS}

Following Cercignani [20], we define an accommodation coefficient $a_{c}(\psi)$ for an arbitrary function $\psi(\xi)$ of the molecular velocity as

$$
a_{c}(\psi)=\frac{(\psi, f)_{-}-(\psi, f)_{+}}{(\psi, f)_{-}-(\psi, M)_{+}},
$$

where $f$ is the VDF on the boundary and

$$
\begin{aligned}
(\psi, \phi)_{-} & =\int_{\xi \cdot n<0}|\boldsymbol{\xi} \cdot \boldsymbol{n}| \psi(\boldsymbol{\xi}) \phi(\boldsymbol{\xi}) d \boldsymbol{\xi} \\
(\psi, \phi)_{+} & =\int_{\boldsymbol{\xi} \cdot \boldsymbol{n}>0}|\boldsymbol{\xi} \cdot \boldsymbol{n}| \psi(\boldsymbol{\xi}) \phi(\boldsymbol{\xi}) d \boldsymbol{\xi} \\
M(\boldsymbol{\xi}) & =\frac{(1, f)_{-}}{2 \pi\left(R T_{w}\right)^{2}} \exp \left(-\frac{\left|\boldsymbol{\xi}-\boldsymbol{V}_{w}\right|^{2}}{2 R T_{w}}\right)
\end{aligned}
$$

Here $\boldsymbol{n}$ is the unit normal vector to the boundary pointing to the gas; $\boldsymbol{V}_{w}$ and $T_{w}$ are, respectively, the velocity and temperature of the boundary $\left(\boldsymbol{V}_{w} \cdot \boldsymbol{n}=0\right.$ is assumed).

\section{APPENDIX B: EXTENSION OF THE MODIFIED MAXWELL-TYPE CONDITION}

The scattering kernel (5) of the modified Maxwell-type condition is expressed as a convex combination of the CL kernel (6):

$$
\begin{aligned}
K_{\mathrm{M}}\left(\bar{\xi}, \xi ; \alpha_{i}, \beta_{i}\right)= & \left(1-\alpha_{i}\right)\left(1-\beta_{i}\right) K_{\mathrm{CL}}(\bar{\xi}, \xi ; 0,0) \\
& +\alpha_{i}\left(1-\beta_{i}\right) K_{\mathrm{CL}}(\bar{\xi}, \xi ; 1,0) \\
& +\left(1-\alpha_{i}\right) \beta_{i} K_{\mathrm{CL}}(\bar{\xi}, \xi ; 0,1) \\
& +\alpha_{i} \beta_{i} K_{\mathrm{CL}}(\bar{\xi}, \xi ; 1,1) .
\end{aligned}
$$

Here $K_{\mathrm{M}}$ (or $K_{\mathrm{CL}}$ ) denotes the kernel of the modified Maxwelltype condition (or the CL condition), and the parameters $T_{i}$ and $V_{i}$ are omitted. Now we extend Eq. (B1) to $\beta_{i} \in(1,2]$, employing the following definition for $\beta_{i}>1$ :

$$
\begin{aligned}
K_{\mathrm{M}}\left(\bar{\xi}, \xi ; \alpha_{i}, \beta_{i}\right)= & \left(1-\alpha_{i}\right)\left(\beta_{i}-1\right) K_{\mathrm{CL}}(\bar{\xi}, \xi ; 0,2) \\
& +\alpha_{i}\left(\beta_{i}-1\right) K_{\mathrm{CL}}(\bar{\xi}, \xi ; 1,2) \\
& +\left(1-\alpha_{i}\right)\left(2-\beta_{i}\right) K_{\mathrm{CL}}(\bar{\xi}, \xi ; 0,1) \\
& +\alpha_{i}\left(2-\beta_{i}\right) K_{\mathrm{CL}}(\bar{\xi}, \xi ; 1,1) .
\end{aligned}
$$

Then, when $\beta_{i}>1$, the backscattering dominates as in the case of the CL condition [see the text below Eq. (7)]. Equation (B2) can also be expressed as

$$
\begin{aligned}
K_{M}\left(\bar{\xi}, \xi ; \alpha_{i}, \beta_{i}\right) \\
=\left[\left(1-\alpha_{i}\right) \delta\left(\xi_{r}+\bar{\xi}_{r}\right)+\alpha_{i} \frac{\left|\xi_{r}\right|}{R T_{i}} \exp \left(-\frac{\xi_{r}^{2}}{2 R T_{i}}\right)\right] \\
\quad \times\left[\left(\beta_{i}-1\right) \delta\left(\xi_{\theta}+\bar{\xi}_{\theta}-2 V_{i}\right) \delta\left(\xi_{z}+\bar{\xi}_{z}\right)\right. \\
\left.\quad+\left(2-\beta_{i}\right) \frac{1}{2 \pi R T_{i}} \exp \left(-\frac{\left(\xi_{\theta}-V_{i}\right)^{2}+\xi_{z}^{2}}{2 R T_{i}}\right)\right] .
\end{aligned}
$$

Using the above definition, we can derive the following boundary condition for the fluid-dynamic equation (8) in the case of $\alpha_{i}=\hat{\alpha}_{i} \epsilon$ and $\beta_{i}=2-\hat{\beta}_{i} \epsilon(\epsilon \ll 1)$ : at $\hat{r}=\hat{r}_{i}$,

$$
\begin{aligned}
U_{i}-u_{\theta 0} & =0 \\
\frac{5}{4} \pi^{1 / 2} \gamma_{2} \frac{d \hat{T}_{0}}{d \hat{r}}+\frac{\hat{\alpha}_{i}+\hat{\beta}_{i}}{2} \hat{\rho}_{0}\left(\hat{T}_{w i}-\hat{T}_{0}\right) n_{i} & =0 .
\end{aligned}
$$


Incidentally, Eqs. (B4a) and (11) [instead of (B4b)] are derived for the CL condition in this case.

\section{APPENDIX C: EXACT SOLUTION OF THE LINEARIZED EQUATION}

The linearized system (15) and (16) can be solved immediately. The flow velocity $u$ is written as

$$
u=\frac{C}{\hat{r}}+D \hat{r}
$$

where $C$ and $D$ are constants given as

$$
\begin{aligned}
& C=\frac{1}{E} \hat{r}_{O} \hat{\beta}_{O} \hat{\beta}_{I} U_{I}, \\
& D=\frac{1}{E \hat{r}_{O}^{2}}\left(2 \pi^{1 / 2} \gamma_{1}^{*}-\hat{r}_{O} \hat{\beta}_{O}\right) \hat{\beta}_{I} U_{I}, \\
& E=\left(\hat{r}_{O}-\frac{1}{\hat{r}_{O}}\right) \hat{\beta}_{O} \hat{\beta}_{I}+2 \pi^{1 / 2} \gamma_{1}^{*}\left(\hat{r}_{O} \hat{\beta}_{O}+\frac{\hat{\beta}_{I}}{\hat{r}_{O}^{2}}\right) .
\end{aligned}
$$

One criterion for the occurrence of the velocity inversion has been derived in Ref. [3] from the above solution as

$$
\max _{\hat{r}}\left(\frac{d u}{d \hat{r}}\right) \geqslant 0 \Rightarrow \hat{\beta}_{O} \leqslant \frac{\pi^{1 / 2} \gamma_{1}^{*}}{\hat{r}_{O}}
$$

This criterion is compared with the numerical results of the BGK model equation in Fig. 10(a). Another more strict criterion may be derived as

$$
u\left(\hat{r}_{O}\right) \geqslant u(1) \Rightarrow \hat{\beta}_{O} \leqslant \frac{2 \pi^{1 / 2} \gamma_{1}^{*}}{\hat{r}_{O}\left(\hat{r}_{O}+1\right)} .
$$

The inequality (14) can also be derived from Eqs. (C1) and (C4).

\section{APPENDIX D: DATA FOR THE COMPUTATION OF THE BGK MODEL EQUATION}

Before the computation, we transform the molecular velocities $\left(\xi_{r}, \xi_{\theta}\right)$ into $(\xi, \psi)$ defined as

$$
\xi=\left(\xi_{r}^{2}+\xi_{\theta}^{2}\right)^{1 / 2}, \quad \psi=\arctan \left(\xi_{\theta} / \xi_{r}\right) .
$$

Further, we eliminate $\xi_{z}$ by introducing appropriate marginal VDFs (by the integration with respect to $\xi_{z}$; see, e.g., Refs. [22,26]). In the computations in Sec. IV, we use 241 nonuniform grid points for $r\left(1 \leqslant r / r_{I} \leqslant 2\right)$, where the minimum interval is $4.3 \times 10^{-5} r_{I}$ on the surface of the cylinders and the maximum $6.5 \times 10^{-3} r_{I}$ at the center of the gap; 145 nonuniform grid points are used for $\xi$ in the range $0 \leqslant \xi \leqslant 6 c_{I}$ $\left[c_{I}=\left(2 R T_{I}\right)^{1 / 2}\right.$, where the minimum and maximum intervals are $2.0 \times 10^{-6} c_{I}$ at $\xi=0$ and $0.12 c_{I}$ at $\xi=6 c_{I}$, respectively; 272 nonuniform grid points are used for $\psi(-\pi<\psi \leqslant \pi)$, where the minimum and maximum intervals are $8.4 \times 10^{-4}$ (at $|\psi|=0, \pi / 2$, and $\pi$ ) and $3.6 \times 10^{-2}$ (at $|\psi|=\pi / 4$ and $3 \pi / 4)$, respectively.
[1] D. Einzel, P. Panzer, and M. Liu, Boundary condition for fluid flow: Curved or rough surfaces, Phys. Rev. Lett. 64, 2269 (1990).

[2] K. W. Tibbs, F. Baras, and A. L. Garcia, Anomalous flow profile due to the curvature effect on slip length, Phys. Rev. E 56, 2282 (1997).

[3] K. Aoki, H. Yoshida, T. Nakanishi, and A. L. Garcia, Inverted velocity profile in the cylindrical Couette flow of a rarefied gas, Phys. Rev. E 68, 016302 (2003).

[4] S. K. Stefanov, R. W. Barber, D. R. Emerson, and J. M. Reese, The critical accommodation coefficient for velocity inversion in rarefied cylindrical Couette flow in the slip and near free-molecular regimes, in Rarefied Gas Dynamics: 25th International Symposium, edited by M. S. Ivanov and A. K. Rebrov (Siberian Branch of the Russian Academy of Sciences, Novosibirsk, 2007), pp. 1146-1151.

[5] R. W. Barber, Y. Sun, X. J. Gu, and D. R. Emerson, Isothermal slip flow over curved surfaces, Vacuum 76, 73 (2004).

[6] D. A. Lockerby, J. M. Reese, D. R. Emerson, and R. W. Barber, Velocity boundary condition at solid walls in rarefied gas calculations, Phys. Rev. E 70, 017303 (2004).

[7] S. Yuhong, R. W. Barber, and D. R. Emerson, Inverted velocity profiles in rarefied cylindrical Couette gas flow and the impact of the accommodation coefficient, Phys. Fluids 17, 047102 (2005).

[8] R. S. Myong, J. M. Reese, R. W. Barber, and D. R. Emerson, Velocity slip in microscale cylindrical Couette flow: The Langmuir model, Phys. Fluids 17, 087105 (2005).
[9] S. Kim, Slip velocity and velocity inversion in a cylindrical Couette flow, Phys. Rev. E 79, 036312 (2009).

[10] N. Dongari, R. W. Barber, D. R. Emerson, S. K. Stefanov, Y. Zhang, and J. M. Reese, The effect of Knudsen layers on rarefied cylindrical Couette gas flows, Microfluid. Nanofluid. 14, 31 (2013).

[11] Y. Jung, Velocity inversion in nanochannel flow, Phys. Rev. E 75, 051203 (2007).

[12] Z. Guo, B. Shi, and C. Zheng, Velocity inversion of micro cylindrical Couette flow: A. lattice Boltzmann study, Comput. Math. Appl. 61, 3519 (2011).

[13] S. K. Dadzie and J. G. Méolans, Anisotropic scattering kernel: Generalized and modified Maxwell boundary conditions, J. Math. Phys. 45, 1804 (2004).

[14] K. Yamamoto, H. Takeuchi, and T. Hyakutake, Characteristics of reflected gas molecules at a solid surface, Phys. Fluids 18, 046103 (2006).

[15] H. Matsumoto and K. Kanamori, Monte Carlo simulation of rarefied gas flow induced by wall temperature gradient, in 28th International Symposium on Rarefied Gas Dynamics 2012, edited by M. Mareschal and A. Santos, AIP Conf. Proc. No. 1501 (AIP, New York, 2012), pp. 661-666.

[16] C. Cercignani and M. Lampis, Kinetic models for gas-surface interactions, Transp. Theory Stat. Phys. 1, 101 (1971).

[17] P. L. Bhatnagar, E. P. Gross, and M. Krook, A. model for collision processes in gases. I. Small amplitude processes in charged and neutral one-component systems, Phys. Rev. 94, 511 (1954). 
[18] P. Welander, On the temperature jump in a rarefied gas, Ark. Fys. 7, 507 (1954).

[19] S. Kosuge, Effect of a boundary condition on the cylindrical Couette flow of a rarefied gas, in Proceedings of the 29th International Symposium on Rarefied Gas Dynamics, edited by J. Fan, AIP Conf. Proc. No. 1628 (AIP, New York, 2014), pp. $423-430$.

[20] C. Cercignani, The Boltzmann Equation and Its Applications (Springer-Verlag, New York, 1988).

[21] Y. Sone, Kinetic Theory and Fluid Dynamics (Birkhäuser, Boston, 2002).

[22] Y. Sone, Molecular Gas Dynamics: Theory, Techniques, and Applications (Birkhäuser, Boston, 2007).

[23] Y. Sone, A note on thermal creep in rarefied gas, J. Phys. Soc. Jpn. 29, 1655 (1970).

[24] Y. Sone and K. Aoki, Slightly rarefied gas flow over a specularly reflecting body, Phys. Fluids 20, 571 (1977).

[25] K. Aoki, T. Inamuro, and Y. Onishi, Slightly rarefied gas flow over a body with small accommodation coefficient, J. Phys. Soc. Jpn. 47, 663 (1979).
[26] H. Sugimoto and Y. Sone, Numerical analysis of steady flows of a gas evaporating from its cylindrical condensed phase on the basis of kinetic theory, Phys. Fluids A 4, 419 (1992).

[27] Y. Sone and S. Takata, Discontinuity of the velocity distribution function in a rarefied gas around a convex body and S. layer at the bottom of the Knudsen layer, Transp. Theory Stat. Phys. 21, 501 (1992).

[28] R. G. Lord, Tangential momentum accommodation coefficients of rare gases on polycrystalline metal surfaces, in Rarefied Gas Dynamics, edited by J. L. Potter (AIAA, New York, 1977), pp. 531-538.

[29] E. Steinheil, W. Scherber, M. Seidl, and H. Rieger, Investigations on the interaction of gases and well-defined solid surfaces with respect to possibilities for reduction of aerodynamic friction and aerothermal heating, in Rarefied Gas Dynamics, edited by J. L. Potter (AIAA, New York, 1977), pp. 589-602.

[30] F. O. Goodman and H. Y. Wachman, Dynamics of Gas-Surface Scattering (Academic, New York, 1976).

[31] Yu. G. Semyonov, S. F. Borisov, and P. E. Suetin, Investigation of heat transfer in rarefied gases over a wide range of Knudsen numbers, Int. J. Heat Mass Transf. 27, 1789 (1984). 